\title{
Contour Grouping with Partial Shape Similarity
}

\author{
Chengqian $\mathrm{Wu}^{1}$, Xiang $\mathrm{Bai}^{1}$, Quannan $\mathrm{Li}^{1}$, Xingwei $\mathrm{Yang}^{2}$, and Wenyu $\mathrm{Liu}^{1}$ \\ ${ }^{1}$ Dept. of Electronics and Information Engineering, \\ Huazhong University of Science and Technology, Wuhan, 430074, P.R. China \\ \{angelwuwan, xiang. bai, truthseeker1985\}@gmail.com, \\ liuwy@mail.hust .edu.cn \\ ${ }^{2}$ Dept. of Computer and Information Sciences, Temple University, Philadelphia \\ xingwei.yang@temple.edu
}

\begin{abstract}
In this paper, a novel algorithm is introduced to group contours from clutter images by integrating high-level information (prior of part segments) and low-level information (paths of segmentations of clutter images). The partial shape similarity between these two levels of information is embedded into the particle filter framework, an effective recursively estimating model. The particles in the framework are modeled as the paths on the edges of segmentation results (Normalized Cuts in this paper). At prediction step, the paths extend along the edges of Normalized Cuts; while, at the update step, the weights of particles update according to their partial shape similarity with priors of the trained contour segments. Successful results are achieved against the noise of the testing image, the inaccuracy of the segmentation result as well as the inexactness of the similarity between the contour segment and edges segmentation. The experimental results also demonstrate robust contour grouping performance in the presence of occlusion and large texture variation within the segmented objects.
\end{abstract}

Keywords: Contour grouping, partial shape similarity, particle filter, Normalized Cuts.

\section{Introduction}

Object detection and recognition is a very important issue in computer vision. But due to the high variability of objects and backgrounds in images, it is still an extremely challenging problem. With the progress in shape representation and recognition 123 , researchers start to use shape information to help detecting and recognizing objects in cluttered images 5619]. Different from the methods based on the shape patches [56], we detect and group the contour of the object by using shape similarity between edge segments extracted from the image and the learned contour parts.

Although partial shape similarity is not a new topic, only a relatively small number of approaches deal with it. From the viewpoint of human perception, it is enough to use part of an object in order to recognize the whole object. For example, although Fig. 1 only shows several part segments, it is easy for us to

T. Wada, F. Huang, and S. Lin (Eds.): PSIVT 2009, LNCS 5414, pp. $167-178,2009$.

(C) Springer-Verlag Berlin Heidelberg 2009 


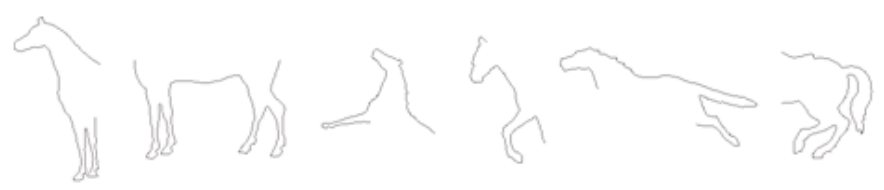

Fig. 1. Parts of the horses

recognize that they represent the contour parts of horses. This example motivates our main hypothesis that contour parts of shapes play an essential role in contour grouping. Based on this hypothesis, our approach is able to group contours of the objects with occlusion or missing parts.

Numerous methods have addressed the detection and contour grouping problems by combining information from different visual levels. Borenstein et al. [13. described a frame that integrates top-down with bottom-up segmentation, in which the fragments are detected in image. Borenstein and Malik [5] introduced a Bayesian model to use shape templates to guide the grouping of the homogenous regions. Recently, Srinivasan and Shi [6] used a fixed parse tree to direct the combination. At each level of the parsing process, the combined mask was measured via shape matching with exemplars. Random field (RF) is used in some method. Tu et al. 17] used data-driven Monte-Carlo sampling to guide generative inference. Levin and Weiss [16] have proposed a CRF based segmentation, emphasizing on combining both top-down and bottom-up learning in loop. Ren et al. 7] gave detailed evaluation performance evaluations for integrating lowlevel, middle-level, high-level cues and a conditional random field formalism is used to combine information. Zheng et al. [8] also combined three levels cues in their method, where classifiers are trained in differently.

Different from the above methods, we learned contour parts instead of shape patches. The partial shape is used as the key information even in the high-level, which is unusual in related works. Besides, we employ particle filtering to integrate the information. As far as we known, it is the very first time that the particle filters is used in such topic.

The first application of particle filter in computer vision is to track the motion boundaries [10. Particle filters have also been used for contour extraction. Piërez et al. [11] applied a sophisticated version of a particle filters model to accomplish the task of contour detection. The approach in 12] uses local symmetry and continuity to group edges to contour parts. The particle filter is extended so that statistical inference based on a reference shape model is possible.

Now we outline the proposed approach. Firstly, for a testing image, we compute its initial segmentation using Normalized Cuts [4. Secondly, we learned the training image to build the database. The database consists of part segments which are classified base on their length percentage. Then, the low-level information from the segmentation of testing image and the high-level information from the database are combined by the framework of particle filter. 


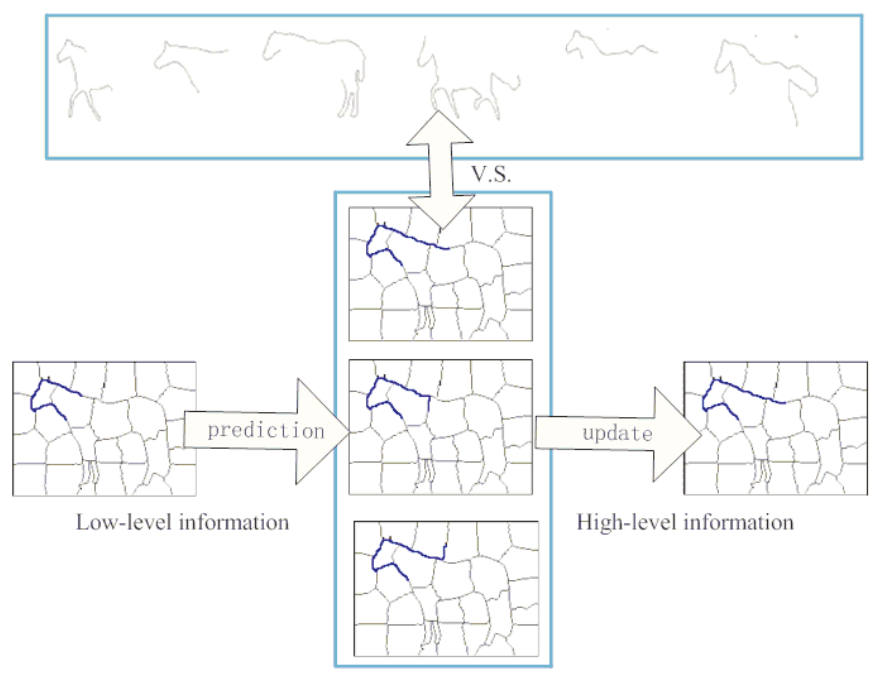

Fig. 2. Outline of particle filter

As the essential step of our method, particle filtering is used to group the object's contour, of which the key idea is to recursively estimate the posterior probability density over the state space conditioned on the data collected so far. Fig. 2 gives the illustration of the process of prediction and updating in particle filters. The blue lines in the Normalized Cuts segmentation images are the paths, which are the particles in our method. At the prediction steps, the paths grow along the edges and generate a group of new paths. At the updating steps, the weights of the newly generated paths updates. As the goal is to find the path that follows the true contour of an object, we define the possibilities (weights) of paths as the partial shape similarity between the paths and the known part segments. Therefore, at the updating steps, the newly generated paths are compared to the part segments in the database, and the new paths' weights are updated based on the partial similarity. Accordingly, the path along the object's contour will be assigned with a higher weight and will be more likely to remain after resampling.

The rest of this paper is organized as follows. Section 2 illustrates the extraction of low-level and high-level information. Section 3 gives the main content of the proposed method, how the particle filters model is used to group contours based on partial shape similarity. Section 4 gives the implementation details and the evaluation of our system followed by Section 5 with conclusion.

\section{Shape Representation}

In this section, we discuss the processes of extracting the low-level information and high-level information. The paths and the part segments are the representations of 
the two levels information respectively. Both of them capture the partial shape of the object, thus the particle filter can combine the two level representations based on the partial similarity.

\subsection{Extraction of the Paths}

The low-level information is obtained from segmentations of the testing image. Normalized Cuts, one of the most popular image segmentation algorithms, is chosen in our method. Fig. 3(b) gives the Normalized Cuts segmentation result of Fig. [3(a).

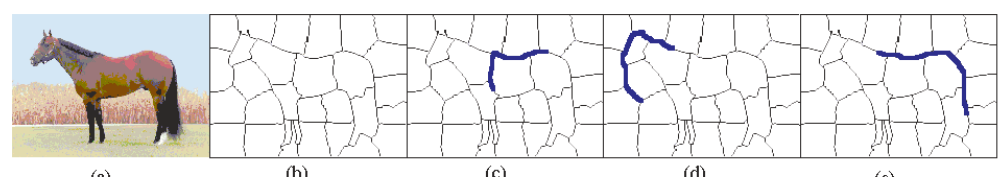

(a)

(b)

(c)

(e)

Fig. 3. (a) Testing image, (b) Normalized Cuts result, (c)-(e) paths (in blue)

Path, the representation of low-level information, is defined as a piece of connected edges from the Normalized Cuts result. Fig. 3(c)-(e) are examples of paths of the testing image. We can observe that some paths (Fig. [3(e)) are along the object's contour; while some are not (Fig. 3(c),(d)).Therefore, the contour grouping method attempts to assign a higher weight to the "correct" path by the particle filter model, so that the algorithm will converge to the object's contour.

Normalization will be applied to the extracted paths, so the comparison between the paths and the part segments is invariant to the planar transformations. This normalized process is the same to the one applied the part segments, of which will be introduced in section 2.2 .

\subsection{Extraction of the Part Segments}

The processes of extraction and description of the high-level information from the training image is illustrated in Fig. 4, Given the contour of the image, firstly, the contour decomposes into a group of part segments, and then a normalization process is applied to the part segments in order to maintain the invariance.

Extraction: Assume that there are $M$ training images, $C=\left(c_{1}, c_{2}, \ldots, c_{M}\right)$ denotes the set of contours of the training images. For each contour $c_{i}(1 \leq i \leq$ $M)$, we sample it into $N$ equidistant points $(N=100)$. The sequence of the sample points of $c_{i}$ is denoted as $S\left(c_{i}\right)=\left(s_{i}^{1}, s_{i}^{2}, \ldots, s_{i}^{N}\right),(1 \leq i \leq M)$, in which $s_{i}^{j}$ means $j^{\text {th }}$ sample points on contour $c_{i}$.

For any pair of sample points $\left(s_{i}^{k}, s_{i}^{l}\right)(1 \leq k, l \leq N ; k \neq l)$ on $c_{i}$, a part segment is obtained by choosing $s_{i}^{k}$ as the start point and $s_{i}^{l}$ as the end point and traversing from the point $s_{i}^{k}$ to the point $s_{i}^{l}$ in clockwise along $c_{i} \cdot s p\left(s_{i}^{k}, s_{i}^{l}\right)$ 


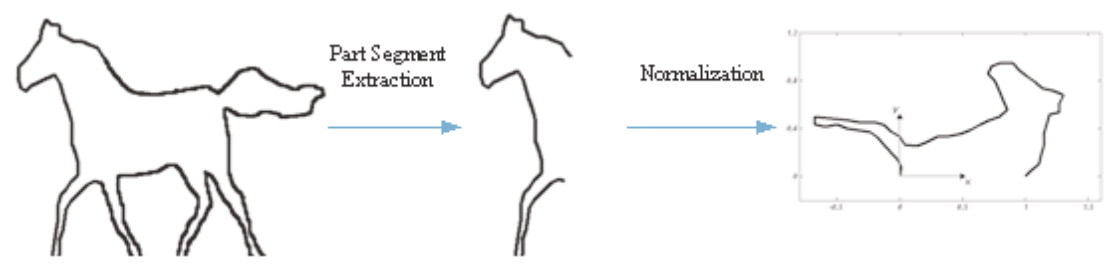

Fig. 4. Extraction processes of the part segments

denotes the part segment. In Fig. 4, a piece of part segment is gotten from contour. By selecting different pair of sample points $\left(s_{i}^{k}, s_{i}^{l}\right)(1 \leq k, l \leq N ; k \neq l)$, a complete set of part segments of contour $c_{i}$ is attained. We use $S P_{i}$ to denote this set of part segments. The part segments set of all the training images is $S P=\left\{S P_{1} \cup S P_{2} \ldots \cup S P_{M}\right\}$.

For each part segment, we compute its length percentage $\operatorname{per}\left(s p\left(s_{i}^{k}, s_{i}^{l}\right)\right)$. Let $L(\cdot)$ be the length function for part segment or a closed contour. The length percentage is computed as $\operatorname{per}\left(s p\left(s_{i}^{k}, s_{i}^{l}\right)\right)=L\left(s p\left(s_{i}^{k}, s_{i}^{l}\right)\right) / L\left(c_{i}\right) \times 100 \%$. The usage of the length percentage will be explained in Section 3.

Normalization: To achieve the invariance to planar transformations (2D translations, rotation, and uniform scaling), we use a similar method in 2] to normalize the part segments.

Firstly, each part segment is resampled with $\mathrm{n}$ equidistant points $(n=50)$. The resampled part segment is denoted as $s p^{\prime}=\left\{x_{1}, x_{2}, \ldots, x_{n}\right\}$, in which $x_{i}$ is a resampled point, $x_{i}=\left(x_{i}, y_{i}\right)(1 \leq i \leq n)$. Then, the resample part segment $s p^{\prime}$ is transformed to the normalized part segment $t p=\left\{x_{1}^{\prime}, x_{2}^{\prime}, \ldots, x_{n}^{\prime}\right\}$. The normalization is realized by mapping $x_{1}$ to $x_{1}^{\prime}=(0,0), x_{n}$ to $x_{n}^{\prime}=(1,0)$ and mapping the remaining points in $s p^{\prime}$ to $x_{2}^{\prime}, \ldots, x_{n-1}^{\prime}$ according to the transformation. The normalized part segment $t p$ is invariant to the $2 \mathrm{D}$ translation, rotation and uniform scaling in the new reference frame. In Fig. 4, the extracted path transforms into the normalized part segment. The normalized (transformed) part segment set for all the training image is denoted as $T P=\left\{T P_{1} \cup T P_{2} \ldots \cup T P_{M}\right\}$. This normalization process is exactly the same to the normalization of paths (Section 2.1).

Building the database: Not all the extracted part segments are used to build the database. Firstly, too short and too long part segments are discarded since they carry little valuable information. In our algorithm, only the part segments with a length percentage that is larger than $20 \%$ and smaller then $80 \%$ are used to build the database. Meanwhile, the part segments that are similar to the linear segment are also abandoned

The part segments in the database are from the same object, horse. We define the classes of the part segments according to the length percentage. $C L_{i}$ denotes the class of part segments which have the length percentage per equals to $i \%$. Therefore, the database updates as $T P=\left\{C L_{20}, C L_{21}, \ldots, C L_{80}\right\}$. The advantage of this classification will be shown in Section 3 . 


\section{Particle Filters Based on Partial Shape Similarity}

The main idea of our method is to combine different levels information using particle filters and update the weights of particles based on the partial shape similarity.

Particle filters (also known as sequential Monte Carlo method) are sophisticated model estimation techniques based on simulation, which aim to estimate the sequence of hidden states $x_{1: k}$ based on the observed data $z_{1: k}$. The commonly used particle filtering algorithm, Sampling Important Resampling (SIR), is chosen in our algorithm, which approximates the filtering distribution $p\left(x_{k} \mid z_{1: k}\right)$ by a weighted set of $N$ particles $\left\{\left(x_{k}^{i}, w_{k}^{i}\right): i=1,2, \ldots, N\right\}$. The main steps for SIR are:

1) Samples from the proposal distribution. The current generation of $\left\{x_{k}^{i}\right\}$ is obtained from the last generation $\left\{x_{k-1}^{i}\right\}$ by sampling from a proposal distribution $\pi\left(x_{k} \mid x_{0: k-1}^{i}, z_{1: k}\right)$.

$$
x_{k}^{i} \sim \pi\left(x_{k} \mid x_{0: k-1}^{i}, z_{1: k}\right)
$$

2) Importance weights: An individual importance weight $\widehat{w}_{k}^{i}$ is assigned to each newly generated particle with the update of the importance weight.

$$
\widehat{w}_{k}^{i} \sim w_{k-1}^{i} \frac{p\left(z_{k}^{i} \mid x_{k}^{i}\right) p\left(x_{k}^{i} \mid x_{k-1}^{i}\right)}{\pi\left(x_{k}^{i} \mid x_{0: k-1}^{i}, z_{1: k}\right)}
$$

The weight $\widehat{w}_{k}^{i}$ is account for the fact that, generally, the proposal distribution $\pi$ is not equal to the true distribution of successor states.

3) Resampling: Particles with a lower importance weight $\widehat{w}_{k}^{i}$ are typically replaced by the samples with a higher weight. This step is necessary since only a finite number of particles are used to approximate a continuous distribution. Furthermore, resampling allows application of particle filter in situations in which the true distribution differs from the proposal.

In our application, the state $x_{k}^{i}$ is a particle represents a piece of path in the testing image. The observation $z_{k}^{i}$ is the likelihood of $x_{k}^{i}$ belonging to the "correct" object's contour. The weights of the particles update according to similarity between the newly generated paths and trained part segments. The paths and the part segments are both partial shape information of the object, and they are embedded with low-level and high-level information respectively. The particle filters algorithm combines different levels of information using the partial shape similarity.

In this section, firstly, we give discussion of our application of particle filters, then we introduce the computation of the partial shape similarity in details.

\subsection{Contour Grouping with Particle Filters}

In this section we firstly introduce the model of the particles and then introduce our application of Sampling Important Resampling (SIR) algorithm. 
The state $x_{k}^{i}=\left\{x p_{k}^{i}, \operatorname{per}^{\prime}\left(x p_{k}^{i}\right)\right\}$ is the $i^{t h}$ particle at the time step $k$, where $x p_{k}^{i}$ denotes the path in the testing image and $\operatorname{per}^{\prime}\left(x p_{k}^{i}\right)$ denotes the length percentage of path $x p_{k}^{i}$. Using cxp denote the object's contour in testing image, the length percentage of path $x p_{k}^{i}$ defined as $\operatorname{per}^{\prime}\left(x p_{k}^{i}\right)=L\left(x p_{k}^{i}\right) / L(c x p) \times 100 \%$, where $L(\cdot)$ is the length function. Length percentage of path $\operatorname{per}^{\prime}\left(x p_{k}^{i}\right)$ is similar to the length percentage of part segments $\operatorname{per}\left(s p\left(s_{i}^{k}, s_{i}^{l}\right)\right)$. It helps to reduce the computation and control the paths' growth at sampling step. Since $\operatorname{cxp}$ is unknown, the above formula is only theoretical one to help understanding. The technical computation of $\mathrm{per}^{\prime}$ will be discussed later.

Sampling process is to obtain the current generation particles $\left\{x_{k}^{i}\right\}$ by sampling from the proposal distribution $\pi\left(x_{k} \mid x_{0: k-1}^{i}, z_{1: k}\right)$. Since the transition prior is easy to draw particles (or samples) and perform subsequent importance weight calculations, it is often used as importance function: $\pi\left(x_{k} \mid x_{0: k}, z_{0: k}\right)=p\left(x_{k} \mid x_{k-1}\right)$. Technically, the sampling process is modeled as the paths grow along the edges of Normalized Cuts result and the growth is controlled in the same speed for each path at every iterative. The definition of the transition prior is

$$
p\left(x_{k}^{i} \mid x_{k-1}^{i}\right)= \begin{cases}\epsilon, & \text { if } x p_{k}^{i} \text { forms a cycle } \\ 1-\epsilon, & L\left(x p_{k}^{i}\right)=L\left(x p_{k-1}^{i}\right) \frac{p r^{\prime}\left(x p_{k-1}^{i}\right)+\Delta p e r}{p e r^{\prime}\left(x p_{k-1}^{i}\right)}\end{cases}
$$

where $\triangle p e r$ is the parameter controlling the growing speed and $\epsilon$ is a very small positive number. The current particles generate as the last generation path grows by a certain length percentage $\triangle p e r$. Besides, the estimated length percentage of $x p_{k}^{i}$ is $\widehat{\operatorname{per}^{\prime}}\left(x p_{k}^{i}\right)=\operatorname{per}^{\prime}\left(x p_{k-1}^{i}\right)+\triangle p e r$. If the path grows through a junction point (see Fig. [5(a), point A), more than one new paths will generate. In Fig. 5 , the path in (a) generates three paths in (b)-(d).

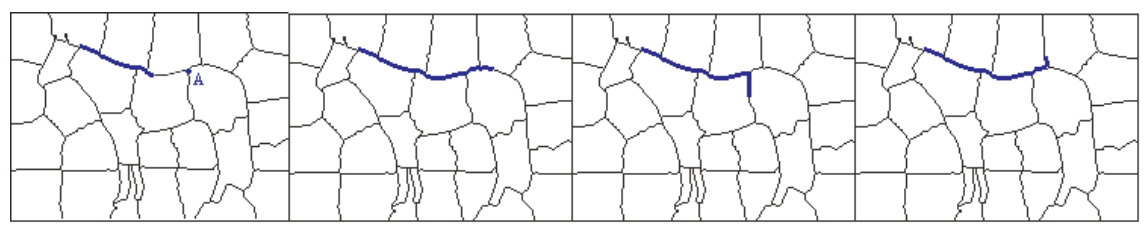

(a) (c)

(d)

Fig. 5. (a) A path, (b)-(d) are three possible extensions of the path in (a)

At the importance weighting step, since the transition prior is used as importance function, formula (2) is rewritten as:

$$
\widehat{w}_{k}^{i} \sim w_{k-1}^{i} \frac{p\left(z_{k}^{i} \mid x_{k}^{i}\right) p\left(x_{k}^{i} \mid x_{k-1}^{i}\right)}{\pi\left(x_{k}^{i} \mid x_{0: k-1}^{i}, z_{0: k}\right)}=w_{k-1}^{i} \frac{p\left(z_{k}^{i} \mid x_{k}^{i}\right) p\left(x_{k}^{i} \mid x_{k-1}^{i}\right)}{p\left(x_{k}^{i} \mid x_{k-1}^{i}\right)}=w_{k-1}^{i} p\left(z_{k}^{i} \mid x_{k}^{i}\right)
$$

We defined the likelihood $p\left(z_{k}^{i} \mid x_{k}^{i}\right)$ as the similarity between the path $x p_{k}^{i}$ and the part segments in training database. It is unnecessary to compare the path 
with the entire database. So, we only compare with those part segments whose length percentage is close to the path's estimated length percentage $\widehat{\operatorname{per}^{\prime}}\left(x p_{k}^{i}\right)$. Therefore, the likelihood $p\left(z_{k}^{i} \mid x_{k}^{i}\right)$ is:

$$
p\left(z_{k}^{i} \mid x_{k}^{i}\right)=p\left(\cup_{j=\widehat{p e r^{\prime}}\left(x p_{k}^{i}\right)-\omega}^{\widehat{\operatorname{per}^{\prime}}\left(x p_{k}^{i}\right)+\omega} C L_{j} \mid x p_{k}^{i}\right)=\underbrace{\widehat{\operatorname{per}^{\prime}}\left(x p_{k}^{i}\right)+\omega}_{j=\widehat{p r^{\prime}}\left(x p_{k}^{i}\right)-\omega} p\left(C L_{j} \mid x p_{k}^{i}\right)
$$

where $\omega$ is an integer parameter controlling the length estimation tolerance. $C L_{j}$ denotes the class of part segments with the length percentage as $j \%$ (Section 2.2). $p\left(C L_{j} \mid x p_{k}^{i}\right)$ is regarded as the similarity between the path and the part segments in $C L_{j}$.

With the likelihood, the particles' weights update. Besides, the length percentages of the paths update as well. The updated length percentage of the path $x p_{k}^{i}$ is computed as:

$$
\operatorname{per}^{\prime}\left(x p_{k}^{i}\right)=\operatorname{argmax}_{j=\widehat{p e r^{\prime}}\left(x p p_{k}^{i}\right)-\omega, \ldots, \widehat{\operatorname{per}^{\prime}}\left(x p_{k}^{i}\right)+\omega} p\left(C L_{j} \mid x p_{k}^{i}\right)
$$

At the resampling step, particles with a lower importance weight are typically replaced by the samples with a higher weight. In our algorithm, we keep the $N_{0}$ particles with highest importance weight. The weights are normalized so that the sum of all the particles is 1 .

\subsection{Computation of Partial Shape Similarity}

We introduce the computation of partial shape similarity in this section. The posterior probability $p\left(C L_{j} \mid x p_{k}^{i}\right)$, the key item in particle filers,interprets as the similarity between the path $x p_{k}^{i}$ and the part segments in $C L_{j}$.

According to the Bayesian rule, the posterior probability of $p\left(C L_{j} \mid x p_{k}^{i}\right)$ is:

$$
p\left(C L_{j} \mid x p_{k}^{i}\right)=\frac{p\left(x p_{k}^{i} \mid C L_{j}\right) p\left(C L_{j}\right)}{p\left(x p_{k}^{i}\right)}
$$

The probability of path $x p_{k}^{i}$ is computed as:

$$
p\left(x p_{k}^{i}\right)=\sum_{j=\widehat{p e r^{\prime}}\left(x p_{k}^{i}\right)-\omega}^{\widehat{p e r^{\prime}}\left(x p_{k}^{i}\right)+\omega} p\left(x p_{k}^{i} \mid C L_{j}\right) p\left(C L_{j}\right)
$$

The class-conditional probability for the path $x p_{k}^{i}$ given part segment tp belongs to the class $C L_{j}$ is

$$
p\left(x p_{k}^{i} \mid C L_{j}\right)=\sum_{t p \in C L_{j}} p\left(x p_{k}^{i} \mid t p\right) p\left(t p \mid C L_{j}\right)
$$

$p\left(x p_{k}^{i} \mid t p\right)$ denotes the similarity between the path $x p_{k}^{i}$ and the part segment $t p$. We use the function of Gaussian to measure the similarity

$$
p\left(x p_{k}^{i} \mid t p\right)=\frac{\exp \left(-\frac{D\left(x p_{k}^{i}, t p\right)^{2}}{2 \delta^{2}}\right)}{\sqrt{2 \pi \delta}}
$$


where the $D\left(x p_{k}^{i}, t p\right)$ is the distance between $x p_{k}^{i}$ and $t p$, and $\delta$ is experimentally decided. The distance between $x p_{k}^{i}$ and $t p$ is

$$
D\left(x p_{k}^{i}, t p\right)=\sum_{j=1}^{n} d\left(x p_{k}^{i}(j), t p(j)\right)
$$

where $n$ is the number of resampled the points after normalization (Section 2.2).

In above formulas, we assume that all classes are equiprobable, i.e. $p\left(C L_{j}\right)=$ $\frac{1}{2 \omega}$, since, at each iterative, $2 \omega$ classes in the database are used in computation. Also, part segments within a class are equiprobable, i.e. $p\left(t p \mid C L_{j}\right)=\frac{1}{\mid C L_{j}}$.

\section{Implementation and Experiments}

Now we describe our algorithm with details and then give the experimental results.

\subsection{Implementation Details}

The particle filter is initialized by selecting the paths form Normalized Cuts segmentation results of the testing image. Since object's contour segments are more likely to have a higher magnitude of gradient, the paths with higher mean gradient magnitude value are chosen. Meanwhile, the length percentage of the part segments starts at $20 \%$, therefore we extend the selected paths to a certain length so that they are long enough.

We stop the particle filters when the estimated length percentage of the particle $\operatorname{per}^{\prime}\left(x p_{k}^{i}\right)$ grows to the threshold $T_{P}$. Generally, the particle with the highest weight represents a true contour part, but, in experiments, we select the top 10 particles in case of noise.

After we get candidate paths from stop step of particle filters, we apply greedysearch for each path and extend it to form closed contour. All the closed contour are considered as candidate contours. The dissimilarity distances between the candidate contours and the training images are calculated using inner-distance shape context method [3]. The candidate contour with the smallest mean distance is the final result.

In experiments, most results are obtained from Normalized Cuts results with 30 blocks. For images with high texture variation, we use 40 blocks. At every iteration, we resample $n=50$ particles. When particles reach the length percentage of $70 \%$, we stop the algorithm.

\subsection{Experiment Results}

We use the horse dataset provided by Borenstein et al. 13 with 50 images selected to build the part database TP . The average time for one image (30 blocks) is 3 minutes on a computer with $1.8 \mathrm{GHz} \mathrm{CPU}$ and $1.0 \mathrm{~GB}$ memory. We can obtain more accurate results on edge images with a large number of regions; however, the processing time will increase significantly. 


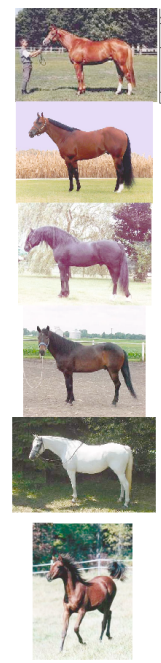

(a)

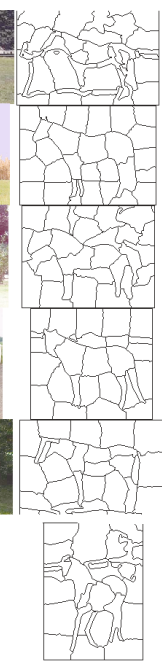

(b)

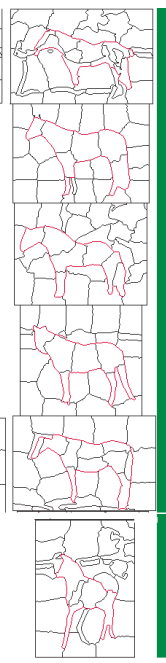

(c)

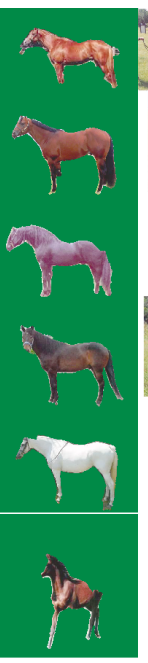

(d)

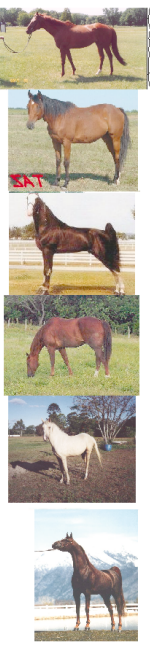

(a)

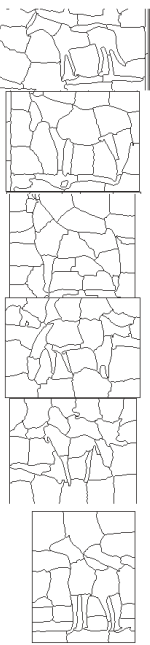

(b)

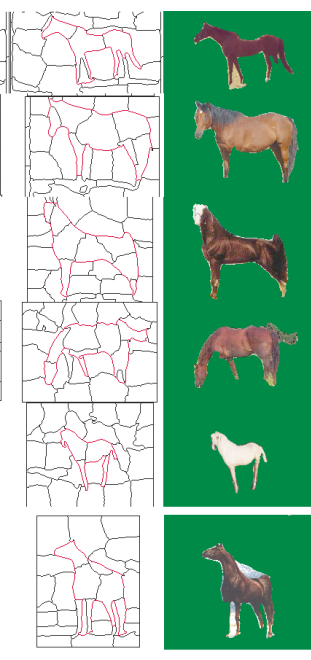

(c)

(d)

Fig. 6. Sample results by our algorithm. (a) are the original input color images, (b) are edge images obtained by Normalized cuts, (c) are the contour grouped (in red) on the edge images(b), and (d) are the detected objects cut from original images.
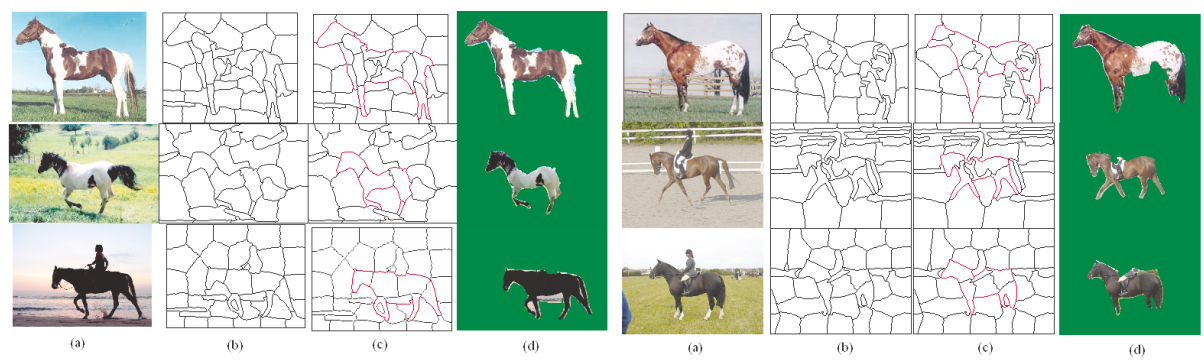

Fig. 7. Sample results on the images with occlusion and large text variation. (a) are the original input images, (b) are edge images obtained by Normalized Cuts, (c) are the contour grouped (in red) on the edge images (b), and (d) are the detected objects.

Performance: Fig. 6 shows some results of our method. We can observe that the detections of the horse are generally successful, although the tail or the legs are missing in some images. We provide a failed result last example in Fig.6.

Experiments on the images with large texture variation or occlusion: Since our method is based on the shape similarity, it performs very well with the presence of occlusion or large texture variations. The results in Fig. 7 prove that our method can obtain very good performance even in the cases of large texture variation or occlusion. (Some of the test images are obtained from Google.) 


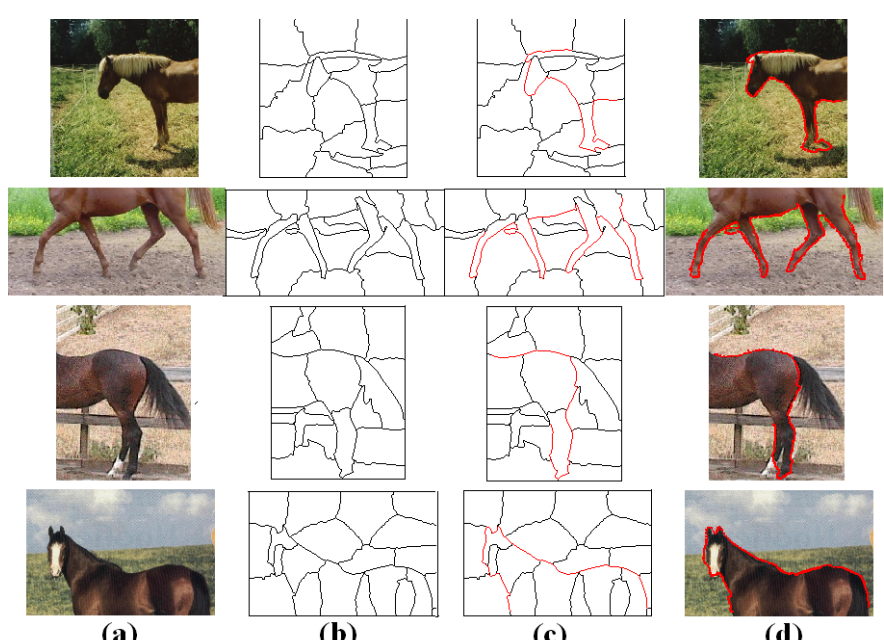

Fig. 8. (a) are the input images, (b) are Normalized Cuts edge images, (c) are the grouped part segments (in red) on (b), and (d) are detected parts on input images

Fig. 8 gives another group of results demonstrate excellent performance of the proposed method against substantial occlusion by cutting the testing images. Although the global shape of the horse is lost, our algorithm still finds the part segment robustly. The methods based the global shape 9141518 are likely to fail on these images, since global information is no longer preserved here.

\section{Conclusion and Future Work}

We proposed a novel contour grouping method based on partial shape similarity. The partial shape representations, paths and part segments, successfully describe the low-level and high-level information, respectively. With the similarity between the paths and part segments, the particle filters combine the different levels of information and group the contour of object in cluttered images. Our method proves that partial shape can be used as the key element for related research fields. The experimental results demonstrate the impressive performance of the method, especially in the cases of large texture variations or occlusions. In the future, we plan to work on: 1) contour grouping using gradient based edges and 2) contour grouping and detection in the case of multiple classes of known shapes.

\section{References}

1. Belongie, S., Malik, J., Puzicha, J.: Shape Matching and Object Recognition Using Shape Contexts. PAMI (2002)

2. Sun, K., Super, B.J.: Classification of Contour Shapes Using Class Segment Sets. In: CVPR (2005) 
3. Ling, H., Jacobs, D.W.: Shape Classification Using the Inner-Distance. PAMI 29(2), 286-299 (2007)

4. Shi, J., Malik, J.: Normalized Cuts and Image Segmentation. In: CVPR (1997)

5. Borenstein, E., Malik, J.: Shape Guided Object Segmentation. In: CVPR (2006)

6. Srinivasan, P., Shi, J.: Bottom-up Recognition and Parsing of the Human Body. In: CVPR (2007)

7. Ren, X., Fowlkes, C., Malik, J.: Cue Integration in Figure/ground Labeling. In: NIPS (2005)

8. Zheng, S., Tu, Z., Yuille, A.: Detecting Object Boundaries Using Low-, Mid-, and High-Level Information. In: CVPR (2007)

9. Kumar, M.P., Torr, P.H.S., Zisserman, A.: OBJ CUT. In: CVPR (2005)

10. Black, M.J., Fleet, D.J.: Probabilistic detection and tracking of motion boundaries. IJCV 38(3), 231-245 (2000)

11. Pérez, P., Blake, A., Gangnet, M.: Jetstream: Probabilistic contour extraction with particles. In: ICCV, pp. 524-531 (2001)

12. Adluru, N., Latecki, L.J., Lakaemper, R., Young, T., Bai, X., Gross, A.: Contour Grouping Based on Local Symmetry. In: ICCV (2007)

13. Borenstein, E., Sharon, E., Ullman, S.: Combining top-down and bottom-up segmentation. In: Proc. IEEE workshop on Perc. Org. in Com. Vis. (2004)

14. McNeill, G., Vijayakumar, S.: Part-based Probabilistic Point Matching Using Equivalence Constraints. In: NIPS (2006)

15. Zöllor, T., Buhumann, J.M.: Robust Image Segmentation Using Resampling and Shape Constraints. PAMI 29(7), 1147-1164 (2007)

16. Levin, A., Weiss, Y.: Learning to combine bottom-up and top-down segmentation. In: Leonardis, A., Bischof, H., Pinz, A. (eds.) ECCV 2006. LNCS, vol. 3954, pp. 581-594. Springer, Heidelberg (2006)

17. Tu, Z., Chen, X., Yuille, A., Zhu, S.C.: Image parsing: unifying segmentation, detection, and object recognition. IJCV (2005)

18. Shotton, J., Blake, A., Cipolla, R.: Contour-Based Learning for Object Detection. In: ICCV (2005)

19. Cremers, D., Kohlberger, T., Schnörr, C.: Shape Statistics in Kernel Space for Variational Image Segmentation. Pattern Recognition 36, 1929-1943 (2003)

20. Tu, Z., Yuille, A.: Shape Matching and Recognition: Using Generative Models and Informative Features. In: Pajdla, T., Matas, J(G.) (eds.) ECCV 2004. LNCS, vol. 3023, pp. 195-209. Springer, Heidelberg (2004) 